\title{
Study on the technology of algin extraction from kelp and preparing edible fresh wrap
}

\author{
Chunhai $\mathrm{Li}^{1, \mathrm{a}}$ \\ ${ }^{1}$ Institute of Light Industry and Food Engineering, Guangxi University, 100 Daxue Eastroad, \\ Nanning 530004, P. R. China \\ agdmm0808@126.com
}

Keywords: Algin; orthogonal experiment; edible fresh wrap

Abstract. The extracting technology of algin from kelp and the preparing technology of edible fresh wrap from algin are reported by this paper. Firstly, the crude algin was extracted from kelp, which was purified by the methods including solid-liquid separation, precipitation, bleaching, transformation, dehydration and etc.. Besides, resulting from the orthogonal experiment, we determined the optimal concentration, time and temperature of digestion. Then, based on the good ductility of algin, we has prepared the edible fresh wrap and explored the different influence factor of preparing the film..

\section{Introduction}

Kelp is a kind of large algae of edible and medicinal value, rich in a variety of organic physiological active substances, such as algin, polysaccharide, etc..[1, 2].Algin is widely used in baking, beverage, candy and food industry due to its special effects of detoxification, anti-cancer, weight loss and etc.. In recent years, edible film as a new type of packaging materials has received much attentions and the application range is becoming more and more widely due to its environment-friendly, biodegradable, harmless and nonpoisonous, high value of nutrition as well as which can enhance the shelf life of food, and improve the quality of food $[3,4]$.

\section{Experiment content and method}

\section{Extraction and purification methods of algin from kelp}

1) Experimental materials and equipments

Kelp, electrothermal constant temperature oven, analytical balance, constant temperature water bath pot, metal mesh

2) Experimental steps

Acid pretreatment, alkali digestion, separation, alginic acid calcium precipitation, bleaching, transformation, dehydration, sol, covering film

3). Calculate the yield

$$
\text { Yield }(\%)=\frac{\text { Weightofproduct }}{\text { Weightofmaterial }} \times 100 \%
$$

\section{Experimental results and discussion}

\section{Extraction of algin}

The result analyzed by one factor optimization shows that concentration, time and temperature are the main factors affected the yield of algin. $\mathrm{NaHCO}_{3}$ concentration between $3 \% \sim 9 \%$, temperature between $55 \sim 65{ }^{\circ} \mathrm{C}$, time between $1 \sim 3$ hours are the scope of three influence factors. 
Table 1 Experiment results $L_{9}\left(3^{3}\right)$

\begin{tabular}{|c|c|c|c|c|}
\hline Level & A & B & $\mathrm{C}$ & $\begin{array}{c}\text { Algin extraction } \\
\text { rate } \%\end{array}$ \\
\hline 1 & 1 & 1 & 1 & 15.1 \\
\hline 2 & 1 & 2 & 2 & 8.9 \\
\hline 3 & 1 & 3 & 3 & 6.6 \\
\hline 4 & 2 & 1 & 2 & 14.3 \\
\hline 5 & 2 & 2 & 3 & 15.6 \\
\hline 6 & 2 & 3 & 1 & 11.6 \\
\hline 7 & 3 & 1 & 3 & 15.2 \\
\hline 8 & 3 & 2 & 1 & 10.7 \\
\hline 9 & 3 & 3 & 2 & 17.2 \\
\hline$K_{1}$ & 30.6 & 44.6 & 37.4 & \\
\hline$K_{2}$ & 41.5 & 35.2 & 40.4 & \\
\hline$K_{3}$ & 43.1 & 35.4 & 37.4 & \\
\hline$k_{1}$ & 10.2 & 14.9 & 12.5 & \\
\hline$k_{2}$ & 13.8 & 11.7 & 13.5 & \\
\hline$k_{3}$ & 14.4 & 11.8 & 12.5 & \\
\hline $\mathrm{R}$ & 4.2 & 3.1 & 1.0 & \\
\hline
\end{tabular}

The orthogonal test indicated that effect order of the three main factors was $\mathrm{A}>\mathrm{B}>\mathrm{C}$. That is to say, the concentration of $\mathrm{NaHCO}_{3}$ was the most influential factor, if the concentration is too low will lead to the decrease of algin extraction rate from kelp. Followed by the temperature of the digestion, which may be lead to decompose of algin at high temperature. Shortening the digestion time under alkali conditions will lead to the result of incomplete digestion, as well prolonging the time will also make the algin decomposition.

Tale 1 indicated that the optimal extraction technology of algin from kelp is $\mathrm{A}_{3} \mathrm{~B}_{1} \mathrm{C}_{2}$ : $9 \%$ concentration of $\mathrm{NaHCO}_{3}$ as digest agent, $55^{\circ} \mathrm{C}$ as the optimal temperature, 2 hours as the best digest time.

The result of the qualitative analysis indicated that the chief ingredient of the extract was algin due to the following reasons: the aqueous solution of extract will result the formation of jelly immediately after adding calcium chloride $\left(\mathrm{CaCl}_{2}\right)$, as well as adding sulfuric acid also generate the insoluble jelly. 


\section{Preparation of edible fresh wrap}

\section{The influence of algin concentration to the film properties}

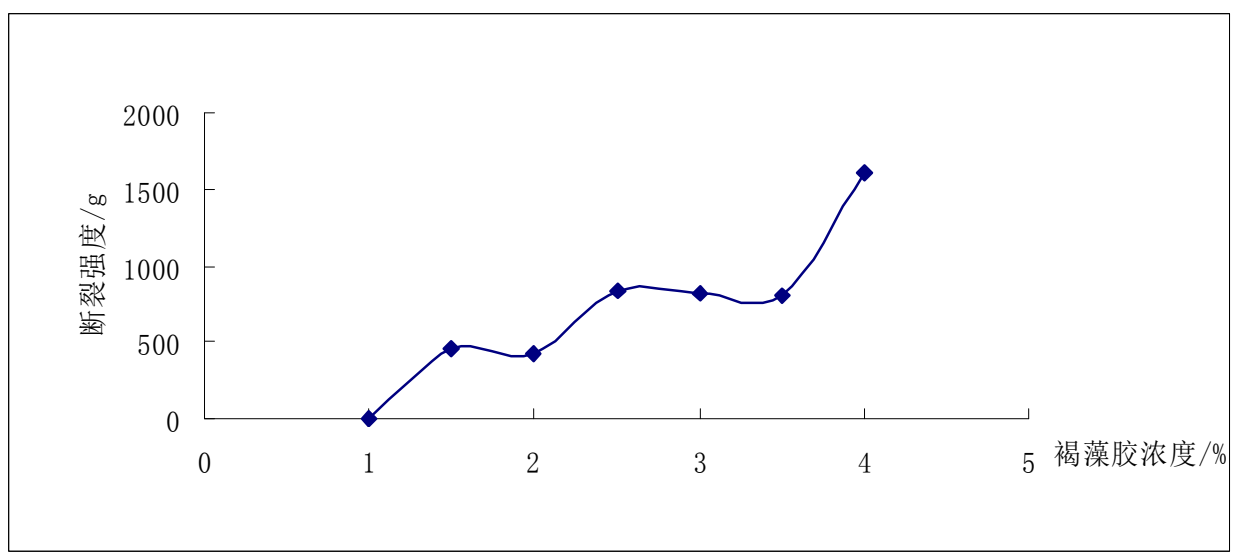

Figure 1. Influence of algin concentration to the film properties

After screening the concentration of algin, we found that $2 \% \sim 3 \%$ is optimal concentration of algin which can get relatively complete transparent film, while below $2 \%$ can not result the whole of the film. Due to the limited solubility of algin in aqueous solution, when dissolved more than $3.5 \%$ of algin in aqueous solution will lead to the film thickness and uneven, and even make the color of the film change to yellow. Therefore, $2.5 \%$ algin solution is appropriate to prepare the film.

\section{The influence of glycerin concentration to the film properties}

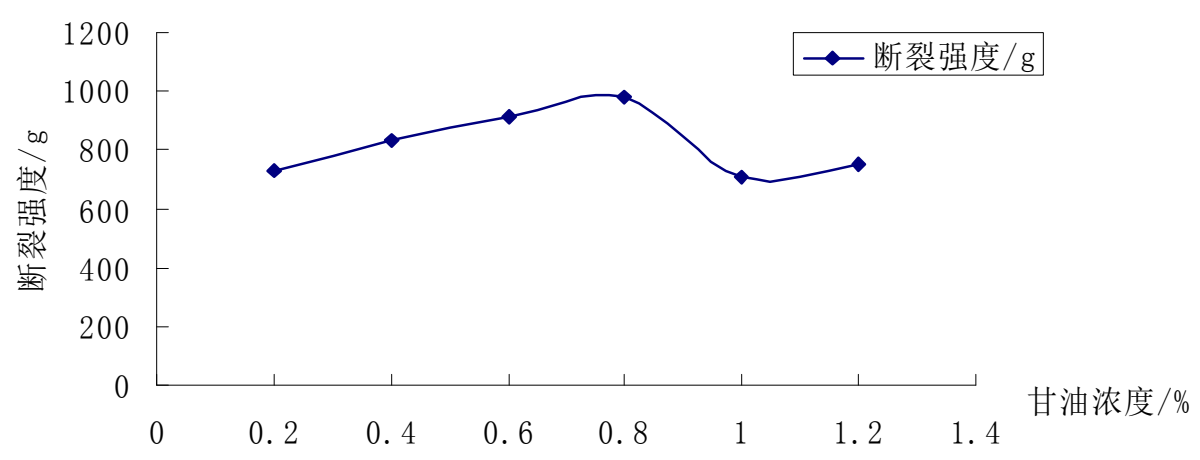

Figure 2. Influence of glycerin concentration to the film properties

Glycerin is a kind of plasticizer, which can increase the flexibility of the thin film and reduce the film tensile strength and brittleness due to it can weaken the intermolecular forces of polysaccharide. Adding glycerin almost does not affect the thickness of the film under a certain concentration, with the increase of concentration of glycerol, accompanying with the increase of film rupture strength, while the concentration of glycerol increasing to a certain degree, fracture strength began to decline. 3.2.3 The determination of calcium chloride solution concentration

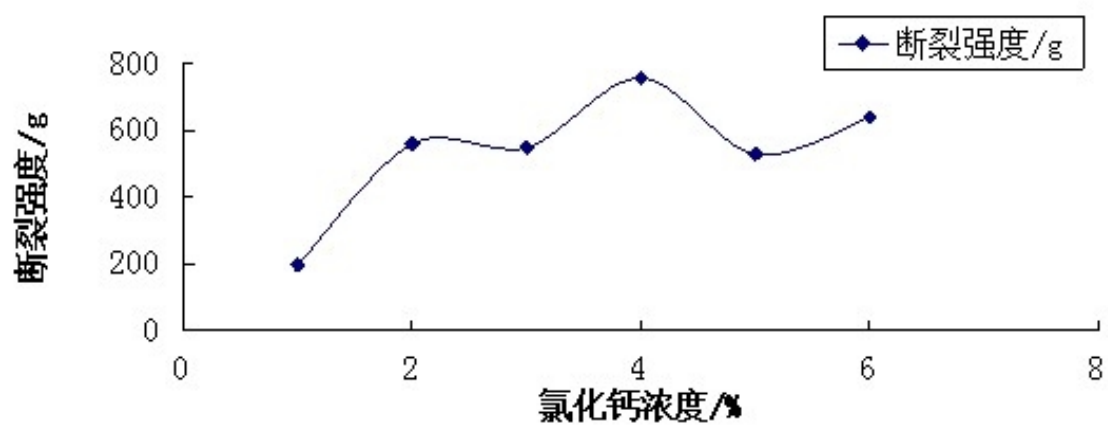

Figure 3 Influence of calcium chloride solution to the rupture strength of the film 
With the concentration increase of $\mathrm{CaCl}_{2}$ solution, the rupture strength increased significantly of the film, when the concentration of $\mathrm{CaCl}_{2}$ was $4 \%$, the fracture strength reached a maximum (757.48 $\mathrm{g}$ ) and then dropped down; As we all know, sodium alginate can complex with with $\mathrm{Ca}^{2}+$ and forms hydrogel, therefore, the cross-linking of $\mathrm{CaCl}_{2}$ with sodium alginate can improve the rupture strength of the film to a certain degree.

\section{Conclusion}

1) The optimal extraction technology of algin from kelp: $9 \%$ concentration of $\mathrm{NaHCO}_{3}$ as digest agent, $55^{\circ} \mathrm{C}$ as the optimal temperature, 2 hours as the best digest time.

2) The best preparation technology of alginate edible film: adding $0.8 \%$ of glycerol to $2.5 \%$ alginate solution and cross-linking 20 min with $4 \%$ of the calcium chloride solution.

\section{References}

[1] L. F Mi, in: Extraction and Purification Technology Research of Laminaria gel, Fujian Agriculture and Forestry University, 2011.

[2] W. R, Gombotz and S. F. Wee: Advanced Drug Delivey Reviews Vol 31(1998), p. 267.

[3] Y. B. Zhang and J. Jiang: China Food Additives Vol 1 (2011), p. 191.

[4] G. R. Chen and Q. Lei: Science and Technologyof Food Indurstry Vol 12 (2011), p. 400. 